\title{
Scalable HPC \& AI Infrastructure for COVID-19 Therapeutics
}

\author{
Hyungro Lee*, Andre Merzky*, Li Tan $₫$, Mikhail Titov*, Matteo Turilli* \\ Dario Alfe ${ }^{\dagger *}$, Agastya Bhati ${ }^{\dagger}$, Alex Brace ${ }^{\S}$, Austin Clyde ${ }^{\dagger}$, Peter Coveney ${ }^{\dagger} \|$, Heng Ma ${ }^{\S}$, \\ Arvind Ramanathan ${ }^{\S}$, Rick Stevens ${ }^{\ddagger \S}$, Anda $\operatorname{Trifan}^{\S}{ }^{\dagger \dagger}$, Hubertus Van Dam ${ }^{\Uparrow}$, \\ Shunzhou $\mathrm{Wan}^{\dagger}$, Sean Wilkinson ${ }^{\ddagger \ddagger}$, Shantenu Jha* 9 \\ ${ }^{*}$ Rutgers University, ${ }^{\top}$ Brookhaven National Laboratory \\ $\dagger$ University College London, ${ }^{\ddagger}$ University of Chicago, ${ }^{\S}$ Argonne National Laboratory, "University of Amsterdam, \\ ** University of Naples Federico II, ${ }^{\dagger \dagger}$ University of Illinois Urbana-Champaign, ${ }^{\ddagger \ddagger}$ Oak Ridge National Laboratory
}

\begin{abstract}
COVID-19 has claimed more $10^{6}$ lives and resulted in over $40 \times 10^{6}$ infections. There is an urgent need to identify drugs that can inhibit SARS-CoV-2. In response, the DOE recently established the Medical Therapeutics project as part of the National Virtual Biotechnology Laboratory, and tasked it with creating the computational infrastructure and methods necessary to advance therapeutics development. We discuss innovations in computational infrastructure and methods that are accelerating and advancing drug design. Specifically, we describe several methods that integrate artificial intelligence and simulation-based approaches, and the design of computational infrastructure to support these methods at scale. We discuss their implementation and characterize their performance, and highlight science advances that these capabilities have enabled.
\end{abstract}

\section{INTRODUCTION}

Considering the universe of about $10^{68}$ possible compounds to traverse for effective drugs, there is an immediate need for more efficient and more effective frameworks for early stage drug discovery [1]. In silico drug discovery is a promising but computationally intensive and complex approach. There is a critical need to improve in silico methodologies to accelerate and select better lead compounds that can proceed to later stages of the drug discovery protocol at the best of times; it is nothing short of a societal and intellectual grand challenge in the age of COVID-19.

A fundamental challenge for in silico drug discovery is the need to cover multiple physical length- and timescales, while spanning an enormous combinatorial and chemical space. In this complex landscape, no single methodological approach can achieve the necessary accuracy of lead compound selection with required computational efficiency. Multiple methodological innovations that accelerate lead compound selections are needed. These methods must in turn, be able to utilize advanced and scalable computational infrastructure.

The primary objective of this paper is to describe the scalable computational infrastructure developed to support innovative methods across diverse and heterogeneous platforms. It discusses four distinct workflows, their computational characteristics, and their implementation using abstractions and RADICAL-Cybertools middleware building blocks. It discusses diverse types of computational capabilities required, the sustained and high watermark performance and scale.
These capabilities provide the computational fabric of the US-DOE National Virtual Biotechnology Laboratory in combination with resources from the EU Centre of Excellence in Computational Biomedicine. To provide a sense of the impact and scale of operations: these methods and infrastructure are being used to screen over 4.2 billion molecules against over a dozen drug targets in SARS-CoV-2. So far, over a 1000 compounds have been identified and experimentally validated, resulting in advanced testing for dozens of hits. The campaign used several million node-hours across diverse HPC platforms, including TACC Frontera, Livermore Computing Lassen, ANL Theta (and associated A100 nodes), LRZ SuperMUC-NG, and ORNL Summit to obtain scientific results.

This paper is organized as follows: In $\S 2$ we outline the computational campaign, and describe the constituent scientific methods and their computational properties. Put together, the collective impact of these methods on drug discovery process is greater than the sum of the individual parts. In $\S 3$ we discuss core middleware building blocks used to develop the computational infrastructure. We describe the design and implementation to support diverse workflows that comprise the campaign over multiple heterogeneous HPC platforms. The following section discusses the performance characteristics and highlights the necessary extensions to overcome challenges of scale and system-software fragility. We conclude in $\$ 5$ with diverse measures of scientific impact towards therapeutic advances by highlighting science results emanating from using these methods and infrastructure for a missionoriented sustained computational campaign.

\section{Computational CAmpaign}

The campaign for discovering new 'hits' (i.e., viable druglike small molecules), and optimizing these hits to viable lead molecules (i.e., that show potential to inhibit viral activity) consists of two iterative loops (Fig. 11). In ensemble docking programs (Sec. [II-A), small molecules are 'docked' against ensembles of conformational states determined from a particular COVID-19 protein target. This is followed by a coarsegrained free-energy estimation (ESMACS-CG) to determine if the docked molecule and the protein target of interest can indeed interact (Sec. II-C). ESMACS-CG samples limited 
relevant conformational states, outputs from ESMACS-CG into ML-driven enhanced sampling methods (Sec. II-B), are taken through a further fine-grained refinement of the binding free-energy (ESMACS-FG; Sec. II-C). The outputs from ESMACS-FG are fed into a machine learning (ML) surrogate for docking, which allows us to quickly estimate whether a given molecule can indeed bind to the COVID-19 protein target. Simultaneously, a secondary iterative loop is developed for a subset of compounds that show promising results from ESMACS-CG, where certain functional groups of promising hits are optimized for protein-target interactions using TIES - a method of lead optimization (Sec. II-D).

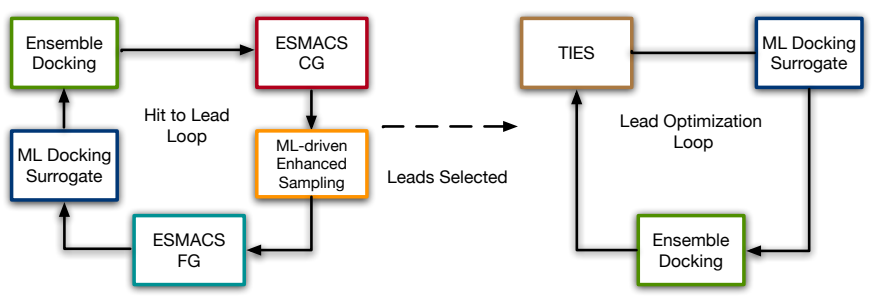

Fig. 1. The computational campaign to advance COVID-19 therapeutics has two coupled loops: drug candidates go through four stages in the Hit-to-Lead loop; a small set of drugs are selected for the Lead Optimization loop. We focus on the following methods: Ensemble Docking, both coarse-grained (CG) and fine-grained (FG) ESMACS, ML-driven Enhanced Sampling and TIES.

\section{A. Computational Protein-Ligand Docking}

Protein-ligand docking is a computational pipeline of ligand 3D structure (conformer) enumeration, exhaustive docking and scoring, and pose scoring. The input requires a protein structure with a designed binding region, or a crystallized ligand from which a region can be inferred, as well as a database of molecules to dock in SMILES format - a compact representation of a 2D molecule.

To take the 2D structures to 3D structures ready for structural docking, proteinization and conformer generation is performed using Omega-Tautomers and, if stereochemistry is not specified, enantiomers are enumerated prior to conformer generation [2]. Typically, tautomers and enantiomers are enumerated for the incoming proposed analog or perturbation to the previous ligand. Conformer generation is performed on the ensemble of structures, generating 200-800 3D conformers for every enantiomer and reasonable tautomer generated. Once the set of 3D structures are enumerated from the 2D smiles, each one is docked against the pocket and scored. The best scoring pose is returned along with its ChemGauss 4 score from exhaustive rigid docking [3].

\section{B. ML-Driven Enhanced Sampling}

Machine learning tools quantify statistical insights into the time-dependent structural changes a biomolecule undergoes in simulations [4], identify events that characterize largescale conformational changes at multiple timescales, build low-dimensional representations of simulation data capturing biophysical or biochemical information, use these lowdimensional representations to infer kinetically and energeti- cally coherent conformational sub-states, and obtain quantitative comparisons with experiments.

Recently, we developed convolutional variational autoencoders (CVAE) that automatically reduce the high dimensionality of MD trajectories and cluster conformations into a small number of conformational states that share similar structural, and energetic characteristics [5]. We apply these approaches on the ESMACS and TIES simulations, outlined shortly. We also used CVAE to drive adaptive simulations for protein folding, and demonstrated that adaptive sampling techniques can provide at least an order of magnitude speedup [6]. These approaches provide acceleration of "rare" events important to study protein-ligand interactions, while leveraging supercomputing platforms [6].

\section{Hit-to-Lead Optimization}

Hit-to-Lead (H2L) is a step in the drug discovery process where promising lead compounds are identified from initial hits generated at preceding stages. It involves evaluation of initial hits followed by some optimization of potentially good compounds to achieve nanomolar affinities. The change in free energy between free and bound states of protein and ligand, also known as binding affinity, is a promising measure of the binding potency of a molecule, and is used as a parameter for evaluating and optimizing hits at $\mathrm{H} 2 \mathrm{~L}$ stage.

We employ the enhanced sampling of molecular dynamics with approximation of continuum solvent (ESMACS) [7] protocol, for estimating binding affinities of protein-ligand complexes. It involves performing an ensemble of molecular dynamics (MD) simulations followed by free energy estimation on the conformations so generated using a semi-empirical method called molecular mechanics Poisson-Boltzmann Surface Area (MMPBSA). The free energies so calculated for the ensemble of conformations are analyzed in a statistically robust manner yielding precise free energy predictions for any given complex. This is particularly important given the fact that the usual practice of performing MMPBSA calculations on conformations generated using a single MD simulation does not give reliable binding affinities. Consequently, ESMACS predictions can be used to rank a large number of hits based on their binding affinities. ESMACS is able to handle large variations in ligand structures, and hence is very suitable for H2L stage where hits have been picked out after covering a substantial region of chemical space. The information and data generated with ESMACS can also be used to train a ML algorithm to improve its predictive capability.

\section{Lead Optimization}

Lead Optimization (LO) is the final step of pre-clinical drug discovery process. It involves altering the structures of selected lead compounds in order to improve their properties such as selectivity, potency and pharmacokinetic parameters. Binding affinity is a useful parameter to make in silico predictions about effects of any chemical alteration in a lead molecule. However, LO requires theoretically more accurate (without much/any approximations) methods for predictions with high 
confidence. In addition, relative binding affinity of pairs of compounds which are structurally similar are of interest, rendering ESMACS unsuitable for LO. We employ thermodynamic integration with enhanced sampling (TIES) [8], which is based on an alchemical free energy method called thermodynamic integration (TI) [9] which fulfill conditions for LO. Alchemical methods involve calculating free energy along a non-physical thermodynamic pathway to get relative free energy between the two end-points. Usually, the alchemical pathway corresponds to transformation of one chemical species into another defined with a coupling parameter $(\lambda)$, ranging between 0 and 1. TIES involves performing an ensemble simulation at each $\lambda$ value to generate the ensemble of conformations to be used for calculating relative free energy. It also involves performing a robust error analysis to yield relative binding affinities with statistically meaningful error bars. The parameters such as the size of the ensemble and the length of simulations are determined keeping in mind the desired level of precision in the results [8].

\section{COMPUTATIONAL INFRASTRUCTURE}

We use the term "task" to indicate a stand-alone process that has well-defined input, output, termination criteria, and dedicated resources. For example, a task can indicate an executable which performs a simulation or a data processing analysis, executing on one or more nodes. A workflow is comprised of tasks with dependencies, whereas a workload represents a set of tasks without dependences or whose dependencies have been resolved. Thus, the tasks of a workload could, resources permitting, be executed concurrently.

\section{A. RADICAL-Cybertools Overview}

RADICAL-Cybertools (RCT) are software systems developed to support the execution of heterogeneous workflows and workloads on one or more high-performance computing (HPC) infrastructures. RCT have three main components: RADICALSAGA (RS) [10], RADICAL-Pilot (RP) [11] and RADICALEnsemble Toolkit (EnTK) [12].

RS is a Python implementation of the Open Grid Forum SAGA standard GFD.90, a high-level interface to distributed infrastructure components like job schedulers, file transfer and resource provisioning services. RS enables interoperability across heterogeneous distributed infrastructures.

$\mathrm{RP}$ is a Python implementation of the pilot paradigm and architectural pattern [13]. Pilot systems enable users to submit pilot jobs to computing infrastructures and then use the resources acquired by the pilot to execute one or more workloads. Tasks are executed concurrently and sequentially, depending on the available resources. RP can execute single or multi core tasks within a single compute node, or across multiple nodes. RP isolates the execution of each tasks into a dedicated process, enabling concurrent execution of heterogeneous tasks. RP offers three unique features compared to other pilot systems on HPC systems: (1) concurrent execution of heterogeneous tasks on the same pilot; (2) support of all the major HPC batch systems; and, (3) support of more than twelve methods to launch tasks.

EnTK is a Python implementation of a workflow engine, designed to support the programming of applications comprised of ensembles of tasks. EnTK executes tasks concurrently or sequentially, depending on their arbitrary priority relation. Tasks are grouped into stages and stages into pipelines depending on the priority relation among tasks. Tasks without reciprocal priority relations can be grouped into the same stage; tasks that need to be executed before other tasks have to be grouped into different stages. Stages are grouped into pipelines and, in turn, multiple pipelines can be executed either concurrently or sequentially. EnTK uses RP, allowing the execution of workflows with heterogeneous tasks.

\section{B. Supporting Multiple Task Execution Modes}

Workflows 1-4 (\$II) have different tasks types and performance requirements that, in turn, require different execution approaches. We discuss three pilot-based task execution frameworks that we developed to support the execution of workflows 1-4. We provide a brief comparison of the three approaches.

1) Execution Mode I: RAPTOR: RP can execute a special type of task, called "worker", that can interpret and execute Python functions. We used this feature to implement a RPbased master/worker framework called RAPTOR (RAdicalPilot Task OveRlay), to distribute multiple Python functions across multiple workers. RAPTOR enables parallel execution of those functions while RP implements capabilities to code both master and worker tasks, and to schedule their execution on the HPC resources acquired by submitting a job.

Fig. 2a illustrates the implementation of RAPTOR on Summit. Once RP has acquired its resources by submiting a job to Summit's batch system, RP bootstraps its Agent (Fig. 2a-1) and launches a task scheduler and a task executor (Fig. 2a. 2). RP Scheduler and RP Executor schedule and launch one or more masters on one the compute nodes (Fig. 2a-3) via either JSRUN [14] or PRRTE [15]. Once running, each master schedules one or more workers on RP Scheduler (Fig. 2a. 4). Those workers are then launched on more compute nodes by RP Executor (Fig. 2a-5). Finally, each master schedules function calls on the available workers for execution (Fig. 2a. 6), load-balancing across workers to obtain maximal resource utilization. The only change needed to use RAPTOR on other machines is a switch of the launch method for the master and worker tasks, e.g., on Frontera, from JSRUN to srun.

2) Execution Mode II: Using multi-DVM: RP supports diverse task launch methods, depending on the availability of specific software systems on the target resources. On Summit, Frontera and Lassen at the Lawrence Livermore National Laboratory, RP supports the use of the Process Management Interface for Exascale (PMIx) and the PMIx Reference RunTime Environment (PRRTE) [15]. PMIx is an open source standard that provides methods to interact with system-level resource managers and process launch mechanisms. PRRTE provides a portable runtime layer that users can leverage to launch a PMIx server. PRRTE includes a persistent mode called Distributed 


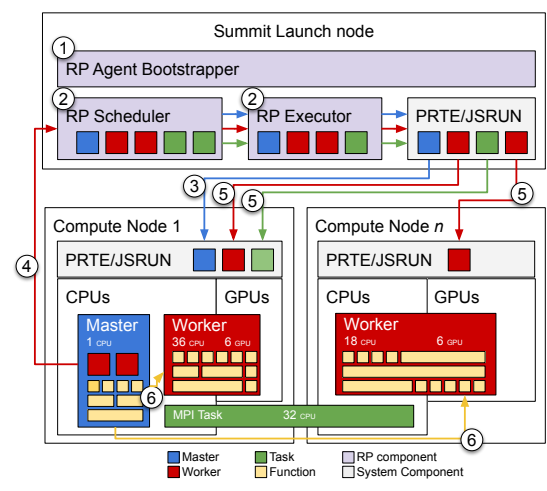

(a)

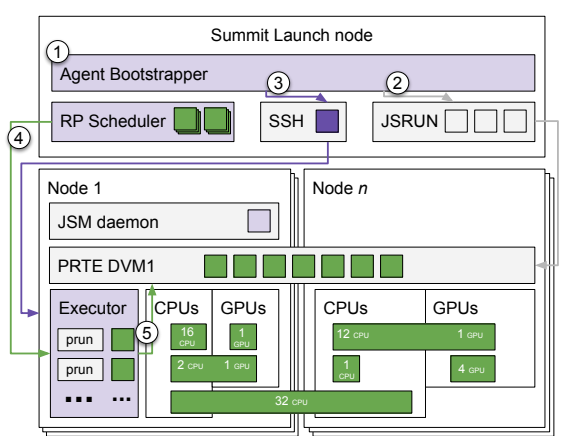

(b)

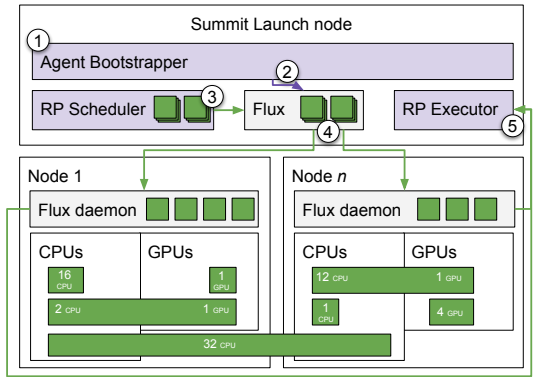

(c)

Fig. 2. Pilot-based task execution frameworks implemented using RADIAL-Pilot.

Virtual Machine (DVM), which uses system-native launch mechanisms to bootstrap an overlay runtime environment, which can be used to launch tasks via the PMIx interface.

One advantage of using PRRTE/PMIx to place and launch stand-alone tasks on thousands of compute nodes, is that they allow for using multiple concurrent DVMs. This enables partitioning of the task execution over multiple, independent sub-systems, reducing the communication and coordination pressure on each sub-system. This improves performance and resilience to PRRTE/PMIx implementation fragility.

Fig. 2b shows the integration between RP and PRRTE/PMIx on Summit. RP bootstraps its Agent (Fig. 2b-1) and, different from the RAPTOR implementation described in Fig. 2a. Agent launches a set of DVMs, each spanning multiple compute nodes (Fig. 2b-2). Agent also uses ssh to execute one or more RP Executor on one or more compute nodes (Fig. 2b-3). Once the DVMs and executors become available, RP schedules tasks on each executor (Fig. 2b-4). Each executor then uses one or more DVMs to place and then launch those tasks (Fig. 2b-5).

3) Execution Mode III: Flux: PRRTE/PMIx introduce a variety of overheads [16]) and their current implementations are still fragile, especially when scheduling more than 20,000 tasks on more than 32 DVMs. Overheads and fragility lead to low resource utilization and unrecoverable failures. For these reasons, RP also supports the use of Flux [17] as an alternative system to schedule, place and launch tasks implemented as stand-alone processes. Fig. $2 \mathrm{c}$ illustrates the integration between RP and Flux. After bootstrapping (Fig. 2c1), RP launches Flux (Fig. 2c-2) and schedules tasks on it for execution (Fig. 2c-3). Flux schedules, places and launches tasks on Summit's compute nodes via its daemons (Fig. 2c-4). RP Executor keeps track of task completion (Fig. 2c-5), and communicates this information to RP Scheduler, based upon which RP Scheduler passes more tasks to Flux for execution.

\section{DeepDriveMD}

To support the requirements of ML-driven enhanced sampling (\$[I-B), we developed DeepDriveMD [6] to employ deep learning techniques, pre-trained models and tuned hyperparameters in conjunction with molecular dynamics (MD) simulations for adaptive sampling. Specifically, DeepDriveMD couples a deep learning (DL) network - called convolutional variational autoencoder (CVAE)) - to multiple MD simulations, to cluster MD trajectories into a small number of conformational states. Insights gained from clustering is used to steer the ensemble MD simulations. This may include either starting new simulations (i.e., expanding the pool of initial MD simulations), or killing unproductive MD simulations (i.e., simulations stuck in metastable states).

DeepDriveMD supports the following computational approach: (1) use an ensemble of MD simulations to generate initial MD data; (2) a 'training' run consisting of a ML algorithm; (3) an 'inference' step where novel starting points for MD are identified; and (4) spawn new MD simulations. DeepDriveMD is built upon EnTK, uses RP for advanced resource management, and is extensible to other learning methods and models, as well as other MD coupling schemes. The current implementation of DeepDriveMD utilizes Tensorflow/Keras (with Horovod for distributed data parallel training) and PyTorch. Typically, a run of DeepDriveMD requires 20 nodes on Summit.

\section{Heterogeneous Task Placement}

Depending on the task launch method, RP places tasks on specific compute nodes, cores and GPU (Figs. 2a and 2b). This placement allows for efficient scheduling of tasks on heterogeneous resources. When scheduling tasks that require different amounts of cores and/or GPUs, RP keeps tracks of the available slots on each compute node of its pilot. Depending on availability, RP schedules MPI tasks within and across compute nodes and reserves a CPU core for each GPU task.

Currently, RP supports four scheduling algorithms: continuous, torus, noop and flux. Continuous is a general purpose algorithm that enables task ordering, task colocation on the same or on different nodes, based on arrival order or explicit task tagging. Torus is a special-purpose algorithm written to support BlueGene architectures, noop allows to pass single or bulk tasks keeping track only of their execution state, and flux delegates scheduling to the Flux framework.

RP opens a large optimization space for specific scheduling algorithms. For example, our continuous scheduler prioritizes tasks that require large amount of cores/GPU so to maximize resource utilization. This could be further extended with explicit clustering or by including information about the execution time of each task. 
These capabilities are used to concurrently execute WF3 and $4(\$[I-C$ and $\$$ II-D), reducing time-to-solution and improving resource utilization at scale. WF3 and WF4 capture ESMACS and TIES respectively, which are both MD-based protocols to compute binding free energies. Both protocols involve multiple stages of equilibration and MD simulations of protein-ligand complexes. Specifically, ESMACS protocol uses the OpenMM MD engine on GPUs, while the TIES protocol uses NAMD on CPUs. Leveraging RP's capabilities, we merge these two "workflows" into an integrated hybrid workflow with heterogeneous tasks which utilize CPU and GPU concurrently.

Fig 3 is a schematic where OpenMM simulations are tasks placed on GPUs, while NAMD simulations are MPI multicore tasks on GPUs. Given that one compute node on Summit has $6 \mathrm{GPU}$ and $42 \mathrm{CPU}$, we are able to run 6 OpenMM tasks in parallel which need $1 \mathrm{GPU}$ and $1 \mathrm{CPU}$ each. For optimal resource utilization, we assign the remaining $36 \mathrm{CPU}$ to 1 NAMD task. NAMD tasks run concurrently on CPU with the OpenMM tasks running on GPU for heterogeneous parallelism (HP). In order to achieve the optimal processor utilization, CPU and GPU computations must overlap as much as possible. We experimentally evaluate heterogeneous parallelism in $\$ \mathrm{IV}-\mathrm{C}$.
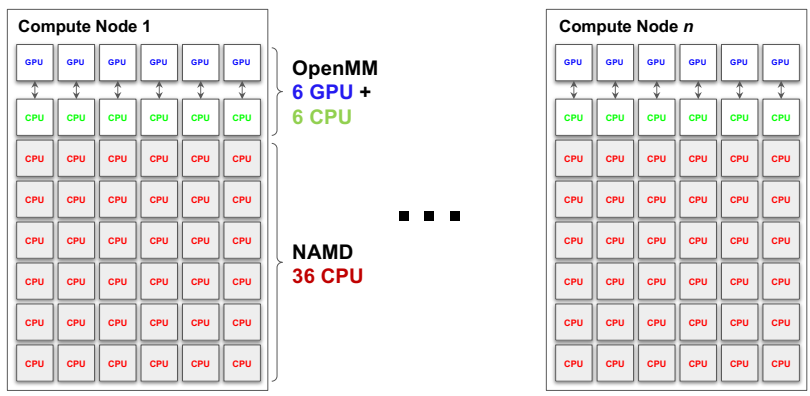

Fig. 3. Using RP's heterogeneous task placement: WF3 is comprised of OpenMM which runs on GPUs; WF4 uses NAMD which runs on CPUs. A hybrid workflow combines WF3 and WF4, concurrently using the CPUs and GPUs of the same Summit node. This increases node utilization significantly.

\section{PERformance Characterization}

\section{A. WF1: Ensemble Consensus Docking}

Compared to physics-based simulation methods, docking is a relatively inexpensive computational process. To increase the reliability of docking results, multiple docking protocols for the same ligand set and protease are preferred over individual docking scores. WF1 uses OpenEye and Autodock-GPU. The former is executed on x86 architectures (e.g., Frontera); the latter on GPUs (e.g., Summit).

For each of the identified receptor sites, WF1 iterates through a list of ligands and computes a docking score for that ligand-receptor pair. The score is written to disk and is used as filter to identify ligands with favorable docking results (score and pose). The docking call is executed as a Python function in OpenEye, and as a self-contained task process in AutoDockGPU. In both cases, the RAPTOR framework (\$III-B1] Fig 2a is used for orchestration.
The duration of the docking computation depends on the type of CPU (OpenEye) or GPU (AutoDock-GPU) used, and the computational requirements of each individual receptor. We measure the docking time (seconds) and docking rate (docks/hr) of three use cases: (1) production runs for NVBLMedical Therapeutics campaigns; and runs for largest achievable size on (2) Frontera and (3) Summit. Table I summarizes the parameterization and results of the experiments we performed for each use case.

WF1 assigns one pilot for each receptor to which a set of ligands will be docked. Within each pilot, one master task is executed for every $\approx 100$ nodes. Each master iterates at different offsets through the ligands database, using precomputed data offsets for faster access, and generating the docking requests to be distributed to the worker tasks. Each worker runs on one node, executing docking requests across the CPU cores/GPUs of that node.

1) Use Case 1: We assigned each of the 31 receptors to a single pilot - that is an independent job submitted to the batch-queue. Due to the different batch-queue waiting times, at most 13 concurrent pilots executed concurrently. With 13way pilot concurrency, the peak throughput was $\approx 17.4 \times$ $10^{6}$ docks/hr. To keep an acceptable load on Frontera's shared filesystem, only 34 of the 56 cores available were used.

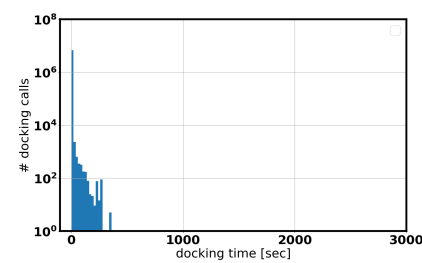

(a)

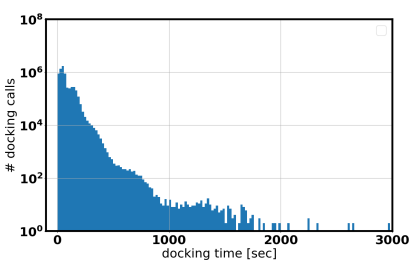

(b)
Fig. 4. WF1, Use Case 1: Distribution of docking runtimes for the receptor with the (a) shortest and (b) longest average docking time out of the 31 receptors analyzed. The distributions of all 31 receptors have a long tail.

Figs. $4 \mathrm{a}$ and $4 \mathrm{~b}$ show the distribution of docking times for receptors with the shortest and longest average docking time, using the Orderable-zinc-ab-enaHLL ligand database. All receptors are characterized by long-tailed docking time distributions. Across the 31 receptors, the $\mathrm{min} / \mathrm{max} / \mathrm{mean}$ docking times are 0.1/3582.6/28.8 seconds (Tab. If), posing a challenge to scalability due to the communication and coordination overheads. The long tail distributions necessitates load balancing across available workers to maximize resource utilization and minimize overall execution time.

We addressed load balancing by: (i) communicating tasks in bulk so as to limit the communication frequency and therefore overhead; (ii) using multiple master processes to limit the number of workers served by each master, avoiding bottlenecks; (iii) using multiple concurrent pilots to partition the docking computations of the set of ligands.

Figs. $5 \mathrm{a}$ and $5 \mathrm{~b}$ show the docking rates for the pilots depicted in Figs. $4 \mathrm{a}$ and $4 \mathrm{~b}$ respectively. As with dock time distributions, the docking rate behavior is similar across receptors. It seems likely that rate fluctuations depend on the interplay 
TABLE I

WF1 USE CASES. FOR EACH USE CASE, RAPTOR USES ONE PILOT FOR EACH RECEPTOR, COMPUTING THE DOCKING SCORE OF A VARIABLE NUMBER OF LIGANDS TO THAT RECEPTOR. OPENEYE AND AUTODOCK-GPU IMPLEMENT DIFFERENT DOCKING ALGORITHMS AND DOCKING SCORES, RESULTING IN DIFFERENT DOCKING TIMES AND RATES. HOWEVER, RESOURCE UTILIZATION IS $>=90 \%$ FOR ALL USE CASES.

\begin{tabular}{|c|c|c|c|c|c|c|c|c|c|c|c|c|}
\hline \multirow{2}{*}{$\begin{array}{l}\text { Use } \\
\text { Case }\end{array}$} & \multirow{2}{*}{ Platform } & \multirow{2}{*}{ Application } & \multirow{2}{*}{ Nodes } & \multirow{2}{*}{ Pilots } & \multirow{2}{*}{$\begin{array}{r}\text { Ligands } \\
{\left[\times 10^{6}\right]}\end{array}$} & \multirow{2}{*}{ Utilization } & \multicolumn{3}{|c|}{ Docking Time [sec] } & \multicolumn{3}{|c|}{ Docking Rate $\left[\times 10^{6}\right.$ docks $\left./ \mathbf{h r}\right]$} \\
\hline & & & & & & & $\min$ & $\max$ & mean & $\min$ & $\max$ & mean \\
\hline 1 & $\mathrm{~F}$ & & 12 & 31 & 205 & $89.6 \%$ & 0. & 3582.6 & 28.8 & 0.2 & 17.4 & 5.0 \\
\hline 2 & Frontera & OpenEye & 3850 & 1 & 125 & $95.5 \%$ & 0.1 & 833.1 & 25.1 & 16.0 & 27.5 & 19.1 \\
\hline 3 & Summit & AutoDock-GPU & 1000 & 1 & 57 & $\approx 95 \%$ & 0.1 & 263.9 & 36.2 & 10.9 & 11.3 & 11.1 \\
\hline
\end{tabular}

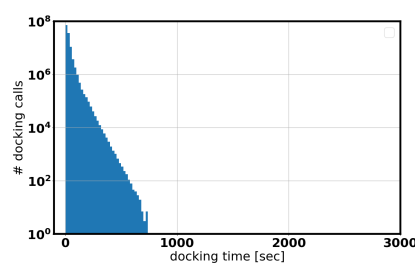

(a)

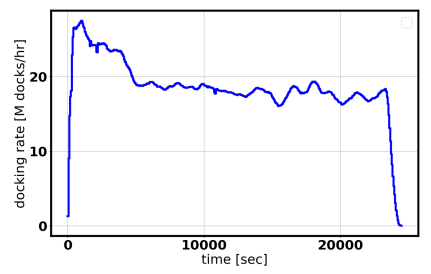

(b)
Fig. 6. WF1, Use Case 2: (a) Distribution of docking time and (b) docking rate for a single receptor and $125 \times 10^{6}$ ligands. Executed with 31 masters, each using $\approx 128$ compute nodes/4352 cores on Frontera.

of machine performance, pilot size, and specific properties of the ligands being docked, and the target receptor. We measure a min/max docking rate of $0.2 / 17.4 \times 10^{6}$ docks/hr with a mean of $5 \times 10^{6}$ docks/hr (Tab. I).

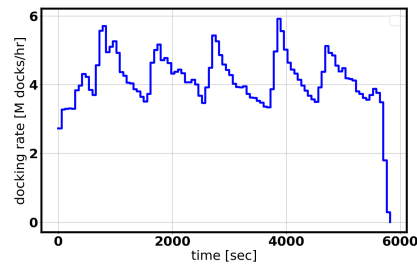

(a)

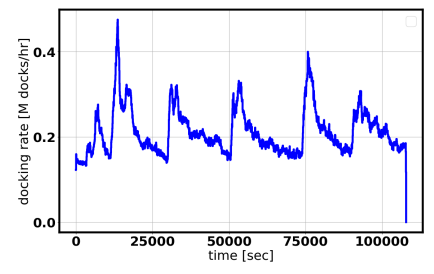

(b)
Fig. 5. WF1, Use Case 1: Docking rates for the receptor with (a) shortest, and (b) longest average docking time.

2) Use Case 2: Fig. 6a shows the distribution of docking times of approximately $125 \times 10^{6}$ ligands from the mcule-ultimate-200204-VJL library to a single receptor using OpenEye on Frontera. The distribution has a min/max of $0.1 / 833.1$ seconds and a mean of 25.1 seconds (Tab. I).

Fig. 6b shows the docking rate for a single pilot with 3850 compute nodes and 130,900 cores. Compared to Use Case 1 , the rate does not fluctuate over time. After peaking at $\approx$ $27.5 \times 10^{6}$ docks $/ \mathrm{hr}$, the rate stabilizes at $\approx 18 \times 10^{6} \mathrm{docks} / \mathrm{hr}$ until the end of the execution (Tab. II). Note that this run was terminated by Frontera's batch system at the end of the given walltime, thus Fig. 6b does not show the "cool down" phase.

Use Case 2 reached $95.5 \%$ core utilization but, as mentioned, we were able to utilize only 34 of the 56 node cores due to filesystem performance limitations.

3) Use Case 3: Figure 7a shows the distribution of the docking times of $\approx 57 \times 10^{6}$ ligands from the mcule-ultimate-200204-VJL database to a single receptor using AutoDock-GPU on Summit. The distribution has a $\mathrm{min} / \mathrm{max} / \mathrm{mean}$ of $0.1 / 263.9 / 36.2$ seconds (Tab. I). The max docking time is shorter than both Use Case 1, Fig. 6a and

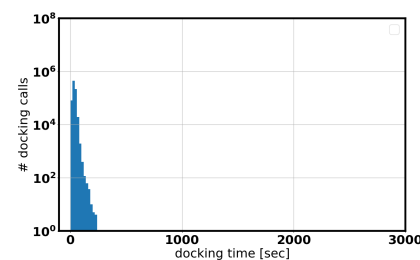

(a)

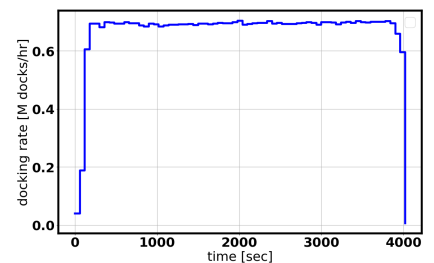

(b)
Fig. 7. WF1, Use Case 3: (a) Distribution of docking time and (b) docking rate for a single receptor and $57 \times 10^{6}$ ligands. Concurrently executed on Summit with a 6000 GPUs pilot.

Use Case 2, Fig. 4, but the mean is longer. As observed, those differences are likely due to specific properties of the docked ligands and the target receptor.

Fig. $7 \mathrm{~b}$ shows the docking rate for a single pilot with 1000 compute nodes, i.e., 6000 GPUs. Different from Use Case 1 and 2 , the rate peaks very rapidly at $\approx 11 \times 10^{6} \mathrm{docks} / \mathrm{hr}$ and maintains that steady rate until the end of the execution. Cool down phase is also very rapid. We do not have enough data to explain the observed sustained dock rate. As with Use Case 2 , we assume an interplay between the scoring function and its implementation in AutoDock-GPU and specific features of the $57 \times 10^{6}$ docked ligands.

Different from OpenEye on Frontera, AutoDock-GPU bundles 16 ligands into one GPU computation in order to efficiently use the GPU memory, reaching an average docking rate of $11.1 \times 10^{6}$ docks $/ \mathrm{hr}$ (Tab. If. Currently, our profiling capabilities allow us to measure GPU utilization with 5\% relative error. Based on our profiling, we utilized between 93 and $98 \%$ of the available GPU resources.

\section{B. WF2: ML-Driven Enhanced Sampling}

WF2 is an iterative pipeline composed of 4 stages. After the first iteration of the 4 stages is completed, if outliers were found, the next iteration starts simulating those outliers; otherwise the simulation continues from where it stopped in the previous iteration. The pipeline stops after a predefined number of iterations.

We measured RCT overhead and resource utilization of WF2 to identify performance bottlenecks. We define RCT overhead as the time spent not executing at least one task. For example, the time spent bootstrapping environments before tasks execution, communicating between EnTK and RabbitMQ (RMQ), or between EnTK and RP while workloads wait to execute. Resource utilization is the percentage of time when resources (CPUs and GPUs) are busy executing tasks. 
The blue bars in Fig. 8 show RCT overheads for the first version of WF2 and how RCT overheads grew with the number of iterations. WF2 may require a variable number of iterations. Thus, our goal was to reduce RCT overhead, and importantly, to make it invariant of the number of iterations.

An initial analysis suggested multiple optimizations of WF2: some of these involved improving the deep learning model and the outlier detection of DeepDriveMD, others required improving RCT. For the latter, we improved the communication protocol between EnTK and RMQ, and we reduced the communication latency between EnTK and RMQ. We avoided sharing connections to RMQ among EnTK threads, reduced the number of concurrent multiple connections and reused communication channels whenever possible.

Fig. 8 (orange) shows the combined effects of improving DeepDriveMD and EnTK communication protocol. Overheads were reduced by $57 \%$ compared to Fig. 8 (blue) but they were still growing with the number of iterations. We moved our RMQ server to Slate, a container orchestration service offered by OLCF. That reduced the communication latency between EnTK and RMQ, as shown in Fig. 8 (green). This allowed RCT overheads to be invariant up to 8 WF2 iterations.

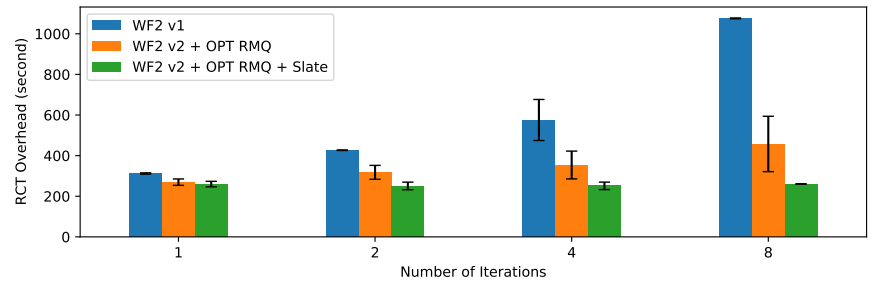

Fig. 8. RCT overhead reduction with improved WF2, EnTK and RabbitMQ.

Fig. 9 depicts resource utilization for different (internal) RCT states as a time-series. "Yellow, light blue and Green space" represents unused resources; "dark" represents resource usage. Fig. 9 shows resource utilization of WF2, when executing four pipeline iterations on Summit with 20, 40, and 80 compute nodes. Note that most of the unused resources are CPU cores that are not needed by WF2. Overall, we measured $91 \%, 91 \%, 89 \%$ GPU utilization respectively. Across scales, Fig. 9 shows differences in the execution time of some of the pipeline stages but no relevant increase of the time spent without executing at least one task.

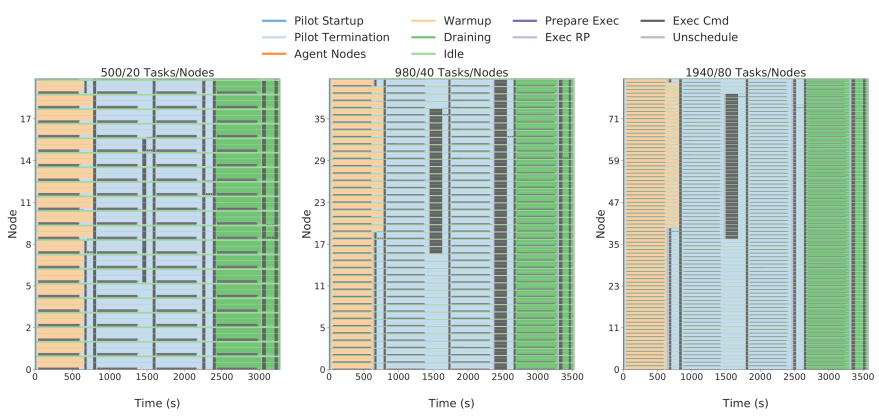

Fig. 9. WF2 Resource Utilization: 20 nodes (left), 40 nodes (center), and 80 nodes (right).

\section{Hybrid Workflows Using Heterogeneous Tasks}

WF3 and WF4 are computationally intensive methods that cost several orders of magnitude more node-hours per ligand than WF1 [18]. As discussed in \$III-D, WF3 and WF4 both compute binding free energies, but have workloads comprised of distinct tasks: GPU-based OpenMM, and CPU-based NAMD tasks respectively. Merging WF3 and WF4 into a single hybrid workflow allowed us to improve resource utilization by employing RP's unique capability of concurrently executing distinct tasks on CPU cores and GPUs. We evaluated that capability by measuring: (i) RCT overhead (as defined previously) as a function of scale; (ii) scalability as a function of problem and resource size; and (iii) resource utilization.

Fig. 10 compares RCT overhead to workflow time to completion (TTX) on 32 nodes for different tasks counts, representing different production workflow configurations. TTX in Fig. 10(c) illustrates concurrent execution of GPU and CPU tasks. The modest increase in TTX compared to Fig. 10(b) is likely due to interference from sharing resources across tasks (Fig. 3), and some scheduler inefficiency. A careful evaluation and optimization will form the basis of further investigation. Fig. 10 d) plots the TTX for the Hybrid-LB scenario when the number of WF3 and WF4 tasks are selected to ensure optimal resource utilization. The number of WF3 tasks completed in Fig. 10 (d) is twice the number of WF3 tasks completed in Fig. 10.c), with no discernible increase in TTX.

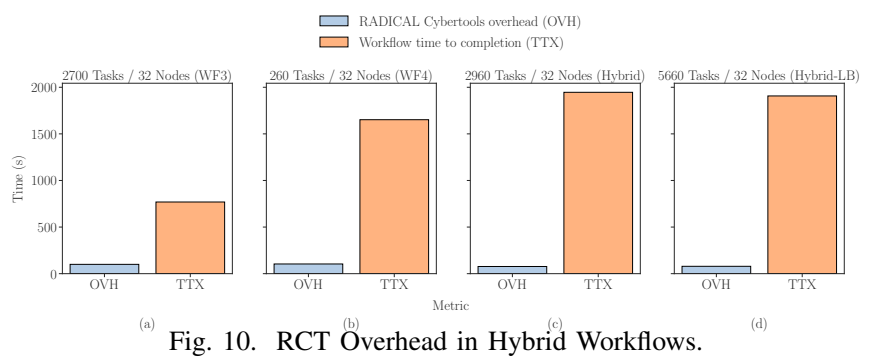

Fig. 11 depicts RCT resource utilization for the configurations of Fig. 10.(c) and Fig. 10/d). As with Fig. 9. "green space" represents unused resources; "dark space" represents resource usage. WF3 and WF4 have 4 and 3 stages respectively, which can be discerned from black bars. Fig. 11 (b) shows greater dark space and thus resource utilization than Fig. 11 (a), representing greater overlap of tasks on GPUs and CPUs due to workload sizing. Both have higher resource utilization than configurations in Fig. 10.a) and (b) due to concurrent CPU and GPU usage.

Fig. 12 shows the scalability of hybrid workflows with load balance enabled and up to 22640 tasks on 128 compute nodes on Summit. The left two panels show the comparison between 5660 GPU tasks and 5660 heterogeneous tasks (5400 GPU tasks $+260 \mathrm{CPU}$ tasks). Note that RCT overhead is invariant between homogeneous and heterogeneous task placements, and with proportionately increasing workloads and node counts (i.e., weak scaling).

In Figs. 10 and 12, RCT overhead varies from 3.8\% to $11.5 \%$ of TTX but it should be noted that task runtimes 


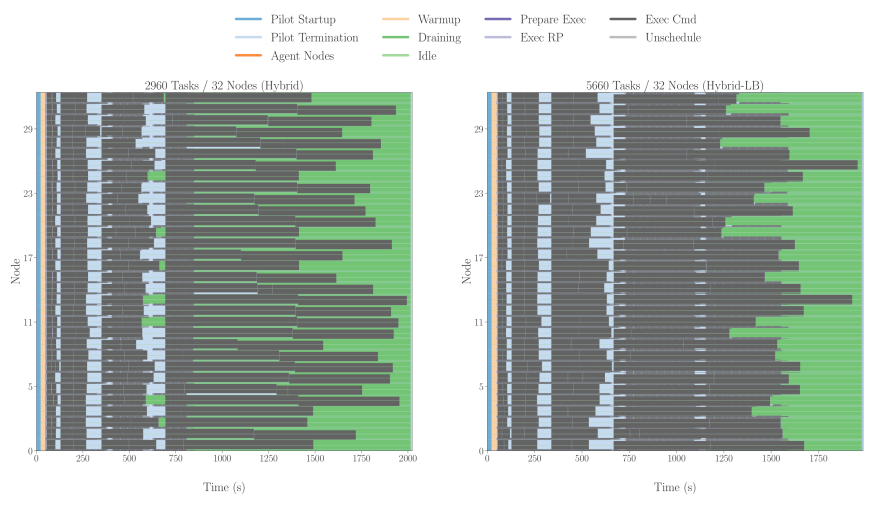

Fig. 11. RCT Resource Utilization in Hybrid Workflows.

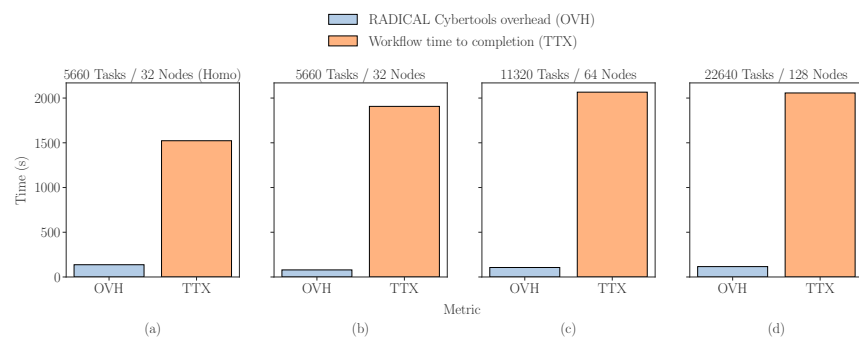

Fig. 12. RCT Overhead in Hybrid Workflows at Scale.

for these experiments are significantly shorter than those of production runs. RCT overhead arises from state transitions and data movements and is essentially invariant of task runtimes, which are reproduced with fidelity in our experiments. Thus, in production runs, RCT overhead is a significantly less proportion of TTX.

Scaling WF3-4: Driven by science results $(\S \mathrm{V})$, and that WF3 \& 4 are the "slowest" per ligand [18], we need to increase the number of nodes and improve reliability across multiple platforms. We preview results for WF3; experience with WF4 and hybrid WF3-4 execution will be reported subsequently.

We performed initial test runs using the multi-DVM execution mode described in \$III-B2, Fig. 2b, and observed that executions were stable with each DVM running on $<50$ nodes and executing $<200$ tasks. Beyond that, we observed interruptions or connectivity losses between executors and DVMs. Further investigation will establish the causes of those limits and possible solutions for higher scalability.

We run WF3-CG on 1000 compute nodes $(+1$ node for RTC), executing 6000 1-GPU tasks on 32 concurrent DVMs. Each DVM spawned $\approx 32$ nodes and executed up to 192 tasks. Fig. 13 shows the utilization of the available resources across different stages of the execution. The pilot startup time (blue) is longer than when using a single DVM [16], mainly due to the 336 seconds spent on launching DVMs which, currently, is a sequential process. Each task requires time to prepare the execution (purple), which mainly includes time for scheduling the task on a DVM, construct the execution command, and process placement and launching by the DVM. The scheduling process takes longer than with a single DVM as it requires to determine which DVM should be used. Further, the construction of the execution command includes a $0.1 \mathrm{~s}$ delay to let
DVM finalize the previous task launching. As each operation is done sequentially per RP executor component, the 0.1 s delay accounts for 600 s alone.

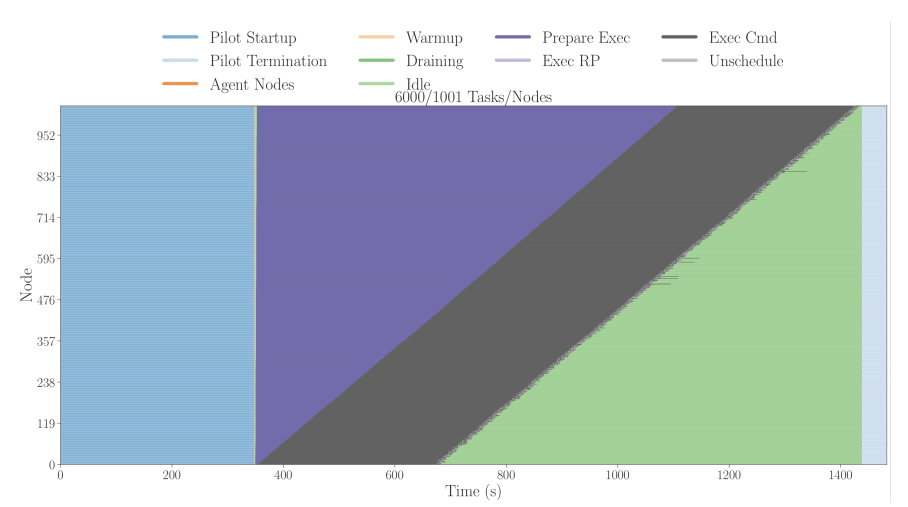

Fig. 13. RCT utilization for WF3-CG using multi-DVM.

As with the other WF3-4 experiments, we reduced task runtimes to limit resource utilization while faithfully reproducing RCT overhead. In Fig. 13, Exec Cmd (task runtime) would be 10 times longer for a production run. Thus, the overheads introduced by using multiple DVMs would have a lesser impact on the overall resource utilization.

We also run WF3-CG on 2000 compute nodes (+1 node for RCT), doubling task and DVM number compared to the run with 1000 nodes. At that scale, we observed three main issues: (i) DVM startup failure; (ii) an internal failure of PRRTE; and (iii) lost DVM connectivity. The majority of tasks were successfully completed (11802 out of 12000), but those issues prevented RCT to gracefully handle their termination.

Given the current fragility of PRRTE/PMIx, we are investigating executing WF3 with the RP/Flux integration described in \$III-B3, Fig. 2c Tab. II summarizes results for our initial test. Performance are comparable to RCT using a single$\mathrm{dvm}$, reducing the overheads measured with the multi-dvm implementation. We experienced no failures, and are now working on deeper integration to further scale our tests. If the current results hold at higher scales, we plan to use the $\mathrm{RP} /$ Flux integration to run the WF3-4 pipeline in production on both Summit and Lassen.

\section{Scientific Results}

The previous section characterized the performance of the scalable HPC and AI infrastructure developed to support campaigns to advance COVID-19 therapeutics. Constituent workflows embody a diverse range of computational characteristics. Tab. III summarizes the heterogeneous platforms utilized, and maps them to specific workflows supported. Put together, the campaign has utilized $2.5 \times 10^{6}$ node-hours on these platforms to support: (i) docking $\sim 10^{11}$ ligands with a peak docking rate of up to $40 \times 10^{6}$ docks/hr; (ii) thousands of AI-driven enhanced sampling simulations; (iii) computed binding free energies on $\sim 10^{5}$ ligand-protein complexes, including $10^{4}$ concurrently. In addition to "raw" scale, individual workflow components demonstrate $100 \times$ to $1000 \times$ scientific improvement over traditional methods. 
TABLE II

WF3 use CASE. TEST RUNS With RP/FLuX INTEGRATION (\$ III-B3 Fig.2C).

\begin{tabular}{llcccccc}
\hline Use Case & Platform & \# Nodes & \# Tasks & \# Failed Tasks & Flux Resource Utilization & Task Scheduling Rate & Execution Time \\
\hline WF3 & Lassen & 128 & 512 & 0 & $88 \%$ & $14.21 \mathrm{t} / \mathrm{s}$ & $6 \mathrm{~m}$ \\
\hline
\end{tabular}

TABLE III

HPC PLATFORMS USED FOR THE COMPUTATIONAL CAMPAIGN. TO MANAGE THE COMPLEXITY ARISING FROM HETEROGENEITY WITHIN AND ACROSS PLATFORMS, REQUIRES MIDDLEWARE ABSTRACTIONS AND DESIGN.

\begin{tabular}{|c|c|c|c|c|c|c|}
\hline HPC Platform & Facility & $\begin{array}{l}\text { Batch } \\
\text { System }\end{array}$ & $\begin{array}{l}\text { Node Architecture } \\
\text { CPU }\end{array}$ & GPU & Workflows & $\begin{array}{r}\text { Max \# nodes } \\
\text { utilized }\end{array}$ \\
\hline & OLCF & LSF & $\mathrm{PO}$ & $x$ Tes & & 2000 \\
\hline Lassen & _NL & LSF & $2 \times$ POWER9 $(2 ?$ & $4 \times$ Tesla V100 & $\mathrm{WF} 2,3$ & 128 \\
\hline Frontera & TACC & Slurm & $2 \times x 86 \_64 \quad(28$ cores $)$ & - & WF1 & 3850 \\
\hline Theta & ALCF & Cobalt & $1 \times$ x86_64 & - & WF1 & 256 \\
\hline SuperMUC-NG & LRZ & Slurm & $2 \times x 86 \_64 \quad(24$ cores $)$ & - & WF3-4 & 6000 (with failures) \\
\hline
\end{tabular}

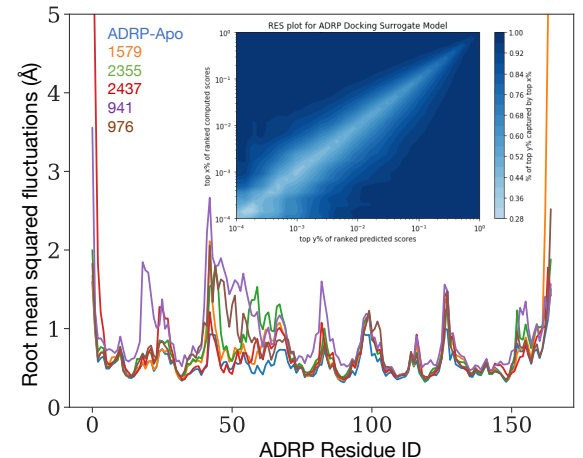

Fig. 14. Root mean square fluctuation of ADRP protein and 5 bound ligands, illustrating that different ligands induce different conformational changes in ADRP. The inset highlights the RES based on the ML-based surrogate model, showing a clear trend in improving the number of downstream calculations needed for finding $n$ compounds in subsequent rounds of the iterative workflow.

The scalable infrastructure provides unprecedented quantitative impact, but also unique qualitative insight, that builds upon information multiple workflows, an example of which we now preview. ML models used to predict docking scores are inherently focused on predicting the ranking of small molecules that potentially bind to and interact stably with the protein target of interest (e.g., ADRP, as presented here). We utilize ML models to accurately rank-order a library of ligands in terms of predicted ranked score, using the regression enrichment surface (RES) technique to examine how well the ML models act as a surrogate for the scoring function [19]. The RES plot (Fig. 14 inset) shows the surrogate model efficiency for detecting true top ranking molecules given a fixed allocation of predicted hits. For instance, if the computing budget allows $n$ number of downstream simulations for inferred molecules of interest, the vertical line representing $n$ on the $x$-axis of the plot shows the fraction of the real top scoring compound distribution captured. Thus, the RES informs the number of top-scoring compounds needed to adequately cover chemical space of ADRP-specific molecules.

Once a potential set of compounds predicted to bind to ADRP are identified using the RES technique, we characterize

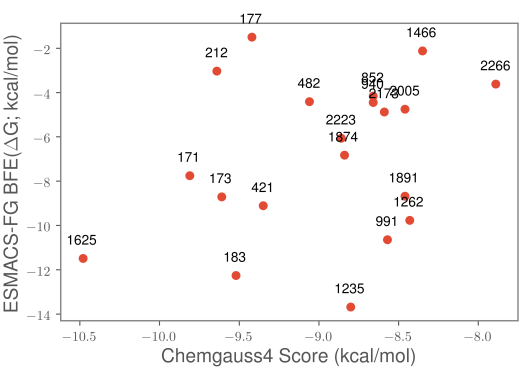

Fig. 15. Correspondence between docking score (ChemGauss4/OpenEye) versus binding free energy using ESMACS-FG for ADRP. Although, the correlation is low, ESMACS-FG acts as an effective filter for compounds that have high affinity to ADRP (e.g., compounds labeled 1625, 183 and 1235.)

how stably they interact with ADRP. We use DeepDriveMD (WF2) to study a small subset of compounds that potentially interact with the primary binding site of ADRP ${ }^{11}$ To characterize the stability, we chose to examine the root mean square fluctuation (RMSF) analysis of the backbone $\mathrm{C}^{\alpha}$ atoms for the apo/ligand-free form of the ADRP protein, which shows decreased fluctuations in the location of its backbone atoms compared to its holo/ ligand-bound counterpart. The ligand-bound protein undergoes higher RMSF fluctuations due to ligand-induced conformational changes, with notable displacements of a few residues such as Asp18-Val20, Asn54Thr67, and His82. While these residues are not in direct contact with the ligand, the binding pocket structural changes causes a ripple effect on downstream residues in the protein.

We then use ESMACS (WF3) to predict which candidates bind tightly (Fig. 15). For instance, compound 1625, has high affinity to ADRP from both ESMACS as well as docking (indicated by the ChemGauss 4 docking score and the ESMACSFG binding free energy values), indicating favorable interactions. TIES (WF4) is used to refine such interactions between the protein and ligand: we performed TIES on 19 compound transformations, which entails mutating the original compound to new ligands with the goal of improving binding affinity. We

\footnotetext{
${ }^{1}$ Although we studied over 200 compounds, we present results from the top five compounds that interact with ADRP stably during $\mathrm{O}(100 \mathrm{~ns})$ simulations
} 
used this method to study the effect of structural changes in a compound on their binding potency with ADRP.

The relative binding affinities $(\Delta \Delta G)$ predicted by TIES for these transformations fall between -0.55 to $4.62 \mathrm{kcal} / \mathrm{mol}$. A positive value indicates a diminished relative binding potency for the "new" compound, whereas a negative value means that the transformation studied is favorable. Twelve out of the 19 transformations studied have $\Delta \Delta G>+1$ which suggests that they all correspond to unfavorable structural changes. The remaining 7 have statistically zero value for $\Delta \Delta G$ which indicates that the corresponding structural modifications do not affect the binding. Both the favorable and unfavorable interactions provide insight into finding compounds with high affinity for target proteins.

\section{DISCUSSION}

Multi-scale biophysics-based computational lead discovery is an important strategy for accelerated drug development. In its current formulation and practice however, it is too inefficient to explore drug compound libraries at the scale of billions of molecules, even on the largest supercomputers. This work demonstrates a path towards improvement by: (i) pairing ML components with, and trained from physics-based components, and (ii) building the HPC and AI infrastructure necessary to enable methodological advances [18]. In doing so, it provides an enhanced drug discovery pipeline for COVID19 - a societal and intellectual grand challenge.

This work is a harbinger of how the role of supercomputers is evolving, to soon become increasingly important "mere generators of data for powerful ML models". This will require the ability to generate data using computational methods that have not historically been used at scale on supercomputers (e.g., WF1). Furthermore, scalable ML-driven methods are needed to improve traditional physics-based approaches [20].

Collectively, supercomputers will increasingly have to support campaigns with diverse components, viz., physics-based simulations, data generation and analysis, and ML/AI tasks. These individual workflows have different computational characteristics and performance challenges. They encompass highthroughput function calls, ensembles of MPI-based simulations, and AI-driven HPC simulations. There are no "turnkey solutions" to support such campaigns across multiple heterogeneous platforms, with the necessary performance and scale to ensure the required throughput. This has necessitated the design, development and iterative improvement of infrastructure to advance therapeutics for COVID-19. The effectiveness and impact of the infrastructure is evidenced by its use to sustain a campaign on multiple heterogeneous platforms over a period of months to generate valuable scientific insight $(\S \mathrm{V})$.

Acknowledgements: Research was supported by the DOE Office of Science through the National Virtual Biotechnology Laboratory; as part of the CANDLE project by the ECP (17-SC-20-SC); UK MRC Medical Bioinformatics project (grant no. MR/L016311/1), UKCOMES (grant no. EP/L00030X/1); EU H2020 CompBioMed2 Centre of Excellence (grant no. 823712), and support from the UCL Provost. Access to SuperMUC-NG (LRZ) was made possible by a special COVID-19 allocation award from the Gauss Centre for Supercomputing in Germany. Anda Trifan acknowledges support from the United States Department of Energy through the Computational Sciences
Graduate Fellowship (DOE CSGF) under grant number: DE-SC0019323. We acknowledge support and allocation from TACC and OLCF.

\section{REFERENCES}

[1] R. S. Bohacek, C. McMartin, and W. C. Guida, "The art and practice of structure-based drug design: A molecular modeling perspective," Medicinal research reviews, vol. 16, no. 1, pp. 3-50, 1996.

[2] "Openeye toolkits 2019.oct.2," Open Eye Scientific, 2019. [Online]. Available: http://www.eyesopen.com/

[3] M. R. Mcgann, H. R. Almond, A. Nicholls, J. A. Grant, and F. K. Brown, "Gaussian docking functions," Biopolymers: Original Research on Biomolecules, vol. 68, no. 1, pp. 76-90, 2003.

[4] G. G. Maisuradze, A. Liwo, and H. A. Scheraga, "Principal component analysis for protein folding dynamics," Journal of Molecular Biology, vol. 385, no. 1, pp. 312 - 329, 2009. [Online]. Available: http://www.sciencedirect.com/science/article/pii/S0022283608012886

[5] D. Bhowmik, S. Gao, M. T. Young, and A. Ramanathan, "Deep clustering of protein folding simulations," BMC Bioinformatics, vol. 19, no. 18 , p. 484, 2018. [Online]. Available: https://doi.org/10.1186/ s12859-018-2507-5

[6] H. Lee, M. Turilli, S. Jha, D. Bhowmik, H. Ma, and A. Ramanathan, "Deepdrivemd: Deep-learning driven adaptive molecular simulations for protein folding," in 2019 IEEE/ACM Third Workshop on Deep Learning on Supercomputers (DLS). IEEE, 2019, pp. 12-19.

[7] S. Wan, A. P. Bhati, S. J. Zasada, I. Wall, D. Green, P. Bamborough, and P. V. Coveney, "Rapid and reliable binding affinity prediction of bromodomain inhibitors: A computational study," Journal of Chemical Theory and Computation, vol. 13, no. 2, pp. 784-795, 2017.

[8] A. P. Bhati, S. Wan, D. W. Wright, and P. V. Coveney, "Rapid, accurate, precise, and reliable relative free energy prediction using ensemble based thermodynamic integration," Journal of Chemical Theory and Computation, vol. 13, no. 1, pp. 210-222, 2017, pMID: 27997169. [Online]. Available: https://doi.org/10.1021/acs.jctc.6b00979

[9] T. P. Straatsma, H. J. C. Berendsen, and J. P. M. Postma, "Free energy of hydrophobic hydration: A molecular dynamics study of noble gases in water," The Journal of Chemical Physics, vol. 85, no. 11, pp. 6720-6727, 1986. [Online]. Available: https://doi.org/10.1063/1.451846

[10] A. Merzky, O. Weidner, and S. Jha, "SAGA: A standardized access layer to heterogeneous distributed computing infrastructure," Software- $X$, vol. $1-2$, pp. 3-8, 2015

[11] A. Merzky, M. Turilli, M. Maldonado, M. Santcroos, and S. Jha, "Using pilot systems to execute many task workloads on supercomputers," in Workshop on Job Scheduling Strategies for Parallel Processing. Springer, 2018, pp. 61-82.

[12] V. Balasubramanian, M. Turilli, W. Hu, M. Lefebvre, W. Lei, R. Modrak, G. Cervone, J. Tromp, and S. Jha, "Harnessing the power of many: Extensible toolkit for scalable ensemble applications," in 2018 IEEE International Parallel and Distributed Processing Symposium (IPDPS). IEEE, 2018, pp. 536-545.

[13] M. Turilli, M. Santcroos, and S. Jha, "A comprehensive perspective on pilot-job systems," ACM Comput. Surv., vol. 51, no. 2, pp. 43:1-43:32, Apr. 2018. [Online]. Available: http://doi.acm.org/10.1145/3177851

[14] D. Quintero, M. Gomez Gonzalez, A. Y. Hussein, and J.-F. Myklebust, IBM High-Performance Computing Insights with IBM Power System AC922 Clustered Solution. IBM Redbooks, 2019.

[15] R. H. Castain, J. Hursey, A. Bouteiller, and D. Solt, "PMIx: Process management for exascale environments," Parallel Computing, vol. 79, pp. 9 - 29, 2018. [Online]. Available: http://www.sciencedirect.com/ science/article/pii/S0167819118302424

[16] M. Turilli, A. Merzky, T. Naughton, W. Elwasif, and S. Jha, "Characterizing the performance of executing many-tasks on summit," IPDRM Workshop, SC19, 2019, https://arxiv.org/abs/1909.03057.

[17] D. H. Ahn, J. Garlick, M. Grondona, D. Lipari, B. Springmeyer, and M. Schulz, "Flux: a next-generation resource management framework for large hpc centers," in 2014 43rd International Conference on Parallel Processing Workshops. IEEE, 2014, pp. 9-17.

[18] A. A. Saadi, D. Alfe, Y. Babuji, A. Bhati, B. Blaiszik, T. Brettin, K. Chard, R. Chard, P. Coveney, A. Trifan et al., "Impeccable: Integrated modeling pipeline for covid cure by assessing better leads," arXiv preprint arXiv:2010.06574, 2020.

[19] A. Clyde, X. Duan, and R. Stevens, "Regression enrichment surfaces: a simple analysis technique for virtual drug screening models," arXiv preprint arXiv:2006.01171, 2020. 
[20] G. Fox and S. Jha, "Learning everywhere: A taxonomy for the integration of machine learning and simulations," IEEE eScience (2019), 2019,
arXiv preprint arXiv:1909.13340. 